of patients admitted and/or staying on different days would be required to delineate the cause(s). Premature attribution to reduced quality of care over the weekend may well run a risk of aggravating the delay in seeking care on the patient side.

0 @ERSpublications

The rhythm of human activity across the week might impact the observed increase in weekend hospital mortality http://ow.ly/KQm2g

Chi Chiu Leung, Lai Bun Tai, Irene Yip and Cheuk Ming Tam

Tuberculosis and Chest Service, Dept of Health, Hong Kong, China.

Correspondence: Chi Chiu Leung, Tuberculosis and Chest Service, Dept of Health, Wan Chai Chest Clinic, 99, Kennedy Road, Wan Chai, Hong Kong, China. E-mail: cc_leung@dh.gov.hk

Received: Feb 022015 | Accepted: Feb 042015

Conflict of interest: None declared.

\title{
References
}

1 Suissa S, Dell'Aniello S, Suissa D, et al. Friday and weekend hospital stays: effects on mortality. Eur Respir J 2014; 44: 627-633.

2 Calverley PM. Something better for the weekend? Eur Respir J 2014; 44: 568-569.

3 Barba R, Zapatero A, Losa JE, et al. The impact of weekends on outcome for acute exacerbations of COPD. Eur Respir J 2012; 39: 46-50.

4 Bell CM, Redelmeier DA. Mortality among patients admitted to hospitals on weekends as compared with weekdays. N Engl J Med 2001; 345: 663-668.

5 Ananthakrishnan AN, McGinley EL, Saeian K. Outcomes of weekend admissions for upper gastrointestinal hemorrhage: a nationwide analysis. Clin Gastroenterol Hepatol 2009; 7: 296-302.

6 Tsoi KK, Chiu PW, Chan FK, et al. The risk of peptic ulcer bleeding mortality in relation to hospital admission on holidays: a cohort study on 8,222 cases of peptic ulcer bleeding. Am J Gastroenterol 2012; 107: 405-410.

7 Voltz R, Kamps R, Greinwald R, et al. Silent night: retrospective database study assessing possibility of "weekend effect" in palliative care. BMJ 2014; 349: g7370.

\section{The emerging role of the contractile and vascular reserves in pulmonary arterial hypertension}

To the Editor:

We read with great interest the recent article by SHARma et al. [1] on the evaluation of right ventricular (RV) reserve in pulmonary arterial hypertension (PAH) by the dobutamine stress test. Their work highlights the potential clinical value of the RV systolic reserve into the evaluation of PAH patients beyond resting measurements. SHARMa et al. [1] reported that RV contractile reserve (tricuspid annular plane systolic excursion (TAPSE) and tricuspid annulus systolic myocardial velocity $\left(S^{\prime}\right)$ ) is markedly attenuated in PAH patients and correlates with exercise capacity. Although the merit of the work is in assessing RV contractile reserve by direct functional parameters, there are some topics that deserve discussion.

Stressors (exercise or pharmacological) of the RV-pulmonary circulation unit in patients with PAH assess the ability of the pathological RV to increase its output (contractile reserve) and of the remodelled pulmonary arterial system to accommodate the flow increase (vascular reserve) [2]. Dobutamine is an ino-chronotropic drug that, at low doses (up to $10-15 \mu \mathrm{g} \cdot \mathrm{kg}^{-1} \cdot \mathrm{min}^{-1}$ ), increases pulmonary blood flow passively without intrinsic vasomotor effects on the pulmonary circulation. The use of higher peak doses of dobutamine could have increased the vasomotor tone of the pulmonary circulation and could explain the significant increase in systolic pulmonary arterial pressure seen in controls [3]. The similar heart rate stress increase in PAH patients compared to controls reported by SHARMA et al. [1] is noteworthy, as it is well known the presence of chronotropic incompetence of PAH patients [4]. 
The majority of the RV systolic function is mediated by longitudinal muscle fibres. So, measures of longitudinal RV shortening reflect the major component of RV systolic function in comparison with measures of transverse/radial function [5]. When considering the measurements of global RV longitudinal function, such as TAPSE, $S^{\prime}$ and myocardial acceleration during isovolumic contraction (IVA), we must be aware of their afterload dependence. IVA appears to be a relatively load-independent estimator of RV contractile response to stress. However, myocardial velocities during the ejection phase are more affected by loading conditions [6]. Although TAPSE has been shown to predict survival in $\mathrm{PAH}$, it is highly dependent on afterload and overall motion of the heart, and fails to reflect RV longitudinal function accurately [7]. This significant afterload dependence could explain the different behaviour of the relationship between change in $(\Delta)$ TAPSE and $\Delta S^{\prime}$ with the peak oxygen uptake [1]. We recently reported that only IVA and $\triangle$ IVA are significantly correlated to cardiac output (CO) and $\triangle \mathrm{CO}$. In contrast, $S^{\prime}$ showed a moderate correlation and TAPSE did not correlate with CO [8]. Besides this, the lower values of TAPSE and $S^{\prime}$ at rest in the PAH group compared with controls, as reported by SHARMA et al. [1], illustrate the significant afterload dependence of TAPSE and $S^{\prime}$, and hence, the inaccurate assessment of the contractile reserve. Load-independent parameters of RV contractility (such as maximal elastance) demonstrated that resting RV contractility is greater than that of healthy controls in virtually all patients with PAH [9]. For this reason, RV dysfunction is best explained by an increase in afterload that is out of proportion to the increase in RV contractility (RV-vascular uncoupling). SHARMA et al. [1] have stated that they avoided IVA due to the chronotropic effects of dobutamine [1]. We disagree with this argument as, in humans, the dependence of heart contractility on increasing heart rate (force-frequency relationship) causes the contractile force to rise as contraction frequency is increased from 60 to $\sim 180$ beats per min.

Both RV function and pulmonary vascular stiffness are determining factors of survival of PAH patients. Hence, the concomitant assessment of pulmonary vascular reserve is very important. A feasible technique for stress testing of the pulmonary vasculature reserve is to assess the mean pulmonary arterial pressure (mPAP)-CO relationship. LAU et al. [10] showed that patients with PAH had an average dobutamine-induced mPAP-CO slope that was significantly higher than in healthy controls, with a markedly reduced distensibility coefficient $\alpha$. Accordingly, we reported a significant increase of the elastic modulus (EM) of the pulmonary artery (PA) in $\mathrm{PAH}$ patients during pharmacological stress, with a negative change of EM in controls [8]. The positive correlation between $\triangle \mathrm{EM}$ and $\triangle \mathrm{mPAP}$ suggests that PA remodelling and buffering function impairment could be associated with the lower vascular reserve of PAH patients. The patients with high vascular reserve (EM and MPAP-CO) and contractile reserve (IVA) had a more preserved haemodynamic response $(\triangle \mathrm{mPAP}<5 \mathrm{mmHg})$ during dobutamine stress testing, and could have a better clinical outcome [8]. However, not every patient with high vascular reserve had a preserved contractile reserve (fig. 1).
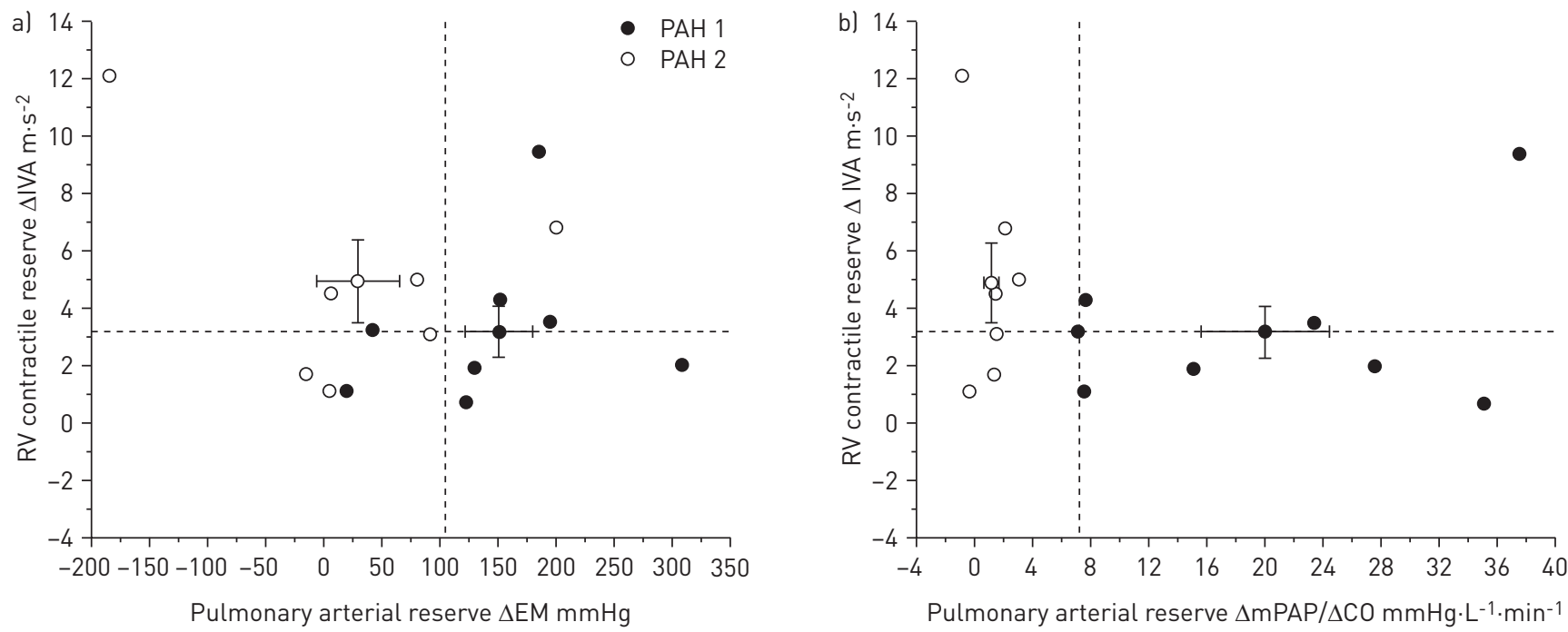

FIGURE 1 Relationship between right ventricular (RV) contractile reserve and pulmonary arterial reserve. Dashed lines represented the median values of $\Delta$ IVA $\left(3.2 \mathrm{~m} \cdot \mathrm{s}^{-2}\right), \Delta \mathrm{EM}(103 \mathrm{mmHg})$ and $\Delta \mathrm{mPAP} / \Delta \mathrm{CO}\left(7 \mathrm{mmHg} \cdot \mathrm{L}^{-1} \cdot \mathrm{min}^{-1}\right) . \Delta$ : dobutamine value minus rest value; IVA: right ventricular myocardial acceleration during isovolumic contraction; EM: pulmonary arterial elastic modulus; mPAP: mean pulmonary arterial pressure; CO: cardiac output; PAH 1: $\triangle$ mPAP $>5 \mathrm{mmHg}$; PAH 2: $\triangle \mathrm{mPAP} \leqslant 5 \mathrm{mmHg}$. 
In conclusion, patients with PAH exhibit markedly diminished RV contractile reserve and pulmonary arterial vascular reserve. Abnormal stress responses occur even in patients with relatively preserved haemodynamics at rest. There is evidence that an impairment of cardiovascular stress response could be correlated with exercise capacity and prognosis. We agree with SHARMA et al. [1] that the potential utility of measuring the cardiovascular reserve and the optimal dosing protocol of dobutamine stress requires further validation studies. vascular reserve http://ow.ly/L7or6

Juan C. Grignola ${ }^{1,2}$ and Enric Domingo ${ }^{3,4}$

${ }^{1}$ Dept of Pathophysiology, Hospital de Clínicas, School of Medicine, Universidad de la República, Montevideo, Uruguay. ${ }^{2}$ Pulmonary Hypertension Unit, Hospital Maciel, Ministerio de Salud Pública, Montevideo, Uruguay. ${ }^{3}$ Cardiologia, Hospital Universitari Vall d'Hebron, Barcelona, Spain. ${ }^{4}$ Dept of Physiology, School of Medicine, Universitat Autonoma de Barcelona, Barcelona, Spain.

Correspondence: Juan C. Grignola, Dept of Pathophysiology, Hospital de Clínicas, School of Medicine, Universidad de la República, Avda Italia 2870, PC 11300 Montevideo, Uruguay. E-mail: jgrig@fmed.edu.uy

Received: Feb 092015 | Accepted: Feb 152015

Conflict of interest: None declared.

\section{References}

1 Sharma T, Lau EMT, Choudhary P, et al. Dobutamine stress for evaluation of right ventricular reserve in pulmonary arterial hypertension. Eur Respir J 2015; 45: 700-708.

2 Haddad F, Vrtovec B, Ashley EA, et al. The concept of ventricular reserve in heart failure and pulmonary hypertension: An old metric that brings us one step closer in our quest for prediction. Curr Opin Cardiol 2011; 26: $123-131$.

3 Vizza CD, Rocca GD, Di Roma A, et al. Acute hemodynamic effects of inhaled NO, dobutamine and a combination of the two in patients with mild to moderate pulmonary hypertension. Crit Care 2011; 5: 355-361.

4 Provencher S, Chemla D, Herve $\mathrm{P}$, et al. Heart rate responses during 6-minute walk test in pulmonary arterial hypertension. Eur Respir J 2006; 27: 114-120.

5 Rudski LG, Lai WW, Afilalo J, et al. Guidelines for the echocardiographic assessment of right heart in adults: a report from the American Society of Echocardiography endorsed by the European Association of Echocardiography, a registered branch of the European Society of Cardiology, and the Canadian Society of Echocardiography. J Am Soc Echocardiogr 2010; 23: 685-713.

6 Vogel M, Schmidt MR, Kristiansen SB, et al. Validation of myocardial acceleration during isovolumic contraction as a novel noninvasive index of right ventricular contractility: comparison with ventricular pressure-volume relations in an animal model. Circulation 2002; 105: 1693-1699.

7 Giusca S, Dambrauskaite V, Scheurwegs C, et al. Deformation imaging describes right ventricular function better than longitudinal displacement of the tricuspid ring. Heart 2010; 96: 281-288.

8 Domingo E, Grignola JC, Aguilar R, et al. Impairment of pulmonary vascular reserve and right ventricular systolic reserve in pulmonary arterial hypertension. BMC Pulm Med 2014; 14: 69.

9 Naeije R, Manes A. The right ventricle in pulmonary arterial hypertension. Eur Respir Rev 2014; 23: 476-487.

10 Lau EM, Vanderpool RR, Choudhary P, et al. Dobutamine stress echocardiography for the assessment of pressure-flow relationships of the pulmonary circulation. Chest 2014; 146: 959-966.

From the authors:

We agree with our colleagues J.C. Grignola and E. Domingo on the importance of measurements of both right ventricular (RV) and pulmonary vascular reserve in patients with pulmonary arterial hypertension, and that further studies are needed to define the most relevant variables and optimal methodology [1]. They have a point that isometric-phase measures of RV systolic function, such as isovolumic myocardial acceleration, may be preferable, as they are less load-dependent than more commonly assessed indices, such as the $S^{\prime}$ wave, tricuspid annular plane systolic excursion, fractional area change or ejection fraction. Isometric-phase measurements best correlate with the gold-standard end-systolic elastance in intact experimental animals [2]. The maximum velocity of isovolumic contraction has been shown to be a stronger predictor of outcome than a number of other echocardiographic measurements of RV function in patients with severe pulmonary hypertension [3]. However, what matters in the end is how RV contractility increases in a fashion adapted to afterload [4]. This may be disclosed by simpler surrogate measurements of contractile reserve such as changes in RV peak systolic pressure during exercise [5]. 